\title{
Gaseous Micropattern Detectors In Astrophysics, Radiology And Plasma Physics
}

\author{
Archana Sharmal \\ University of Maryland, College Park, 20456 MD, USA
}

\begin{abstract}
Multiwire gaseous detectors have matured in the last few decades with major implications in particle physics experiments, they have also been successfully refined for use in other fields: X-rays for medical imaging, UV and single photon detection, neutron and crystal diffraction studies etc. Their major limitation has been a modest rate capability $\left(\right.$ flux $\left.\sim 10^{3} / \mathrm{mm}^{2}\right)$. In the last decade several micropattern position sensitive gas devices have been introduced with an inherently improved rate capability (few $\mathrm{MHz} / \mathrm{mm}^{2}$ ) and a localization accuracy of 40-50 $\mu \mathrm{m}$. They are being extensively pursued for their application in several fields. A state-of-the-art of this new generation of gaseous detectors will be reviewed.
\end{abstract}

\footnotetext{
${ }^{*}$ Invited Plenary Overview Talk at the IEEE Nuclear Science Symposium and Medical Imaging Conference, Lyon, France Oct. 15-20, 2000

${ }^{1}$ Presently at CERN, CH 1211, Geneva, Switzerland. E-mail Archana.Sharma@cern.ch
} 


\title{
Gaseous Micropattern Detectors In Astrophysics, Radiology And Plasma Physics
}

\author{
Archana Sharma ${ }^{2}$ \\ University of Maryland, College Park, 20456 MD, USA
}

\begin{abstract}
Multiwire gaseous detectors have matured in the last few decades with major implications in particle physics experiments, they have also been successfully refined for use in other fields: X-rays for medical imaging, $U V$ and single photon detection, neutron and crystal diffraction studies etc. Their major limitation has been a modest rate capability $\left(\right.$ flux $\sim 10^{3} / \mathrm{mm}^{2}$ ). In the last decade several micropattern position sensitive gas devices have been introduced with an inherently improved rate capability (few $\mathrm{MHz} / \mathrm{mm}^{2}$ ) and a localization accuracy of 40-50 $\mu \mathrm{m}$. They are being extensively pursued for their application in several fields. A state-of-the-art of this new generation of gaseous detectors will be reviewed.
\end{abstract}

\section{INTRODUCTION}

The pioneering work done at the beginning of the nineteenth century by Thomson, Rutherford and Geiger [1] just after the discovery of electromagnetic radiation focussed the attention to developments of 'tools' to detect radiation. The Single Wire Proportional Counter (SWPC) was one of the essential tools in the early part of the last century.

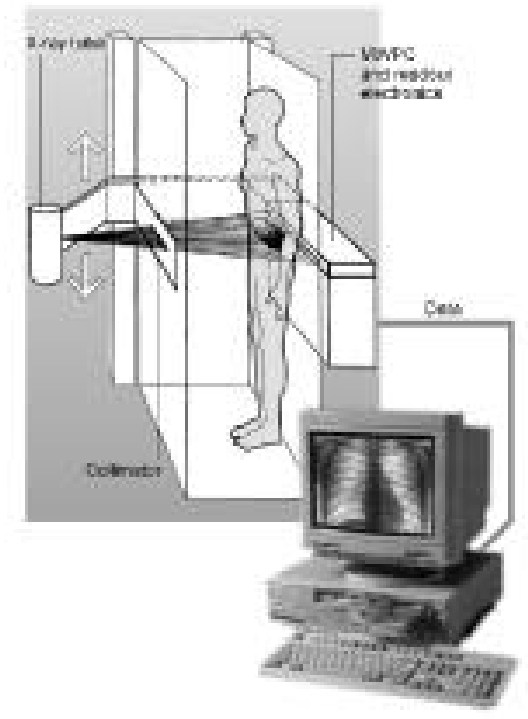

Fig. 1 The Siberian digital radiography device using MWPC [ ]
It was not until the invention of Charpak in 1968 of the Multi-Wire Proportional Chamber (MWPC) [2] that a new era was ushered in this field. The main performance features of the MWPC are a space resolution of few hundred $\mu \mathrm{m}$, two and three-dimensional localization of incident radiation, excellent energy resolution, rate capabilities of a few $\mathrm{kHz} / \mathrm{mm}^{2}$. Two track separations of the order of $2 \mathrm{~mm}$ have been measured and very large active areas and volumes have been effected over the last three decades for charged particle tracking in medium and high energy physics. Several excellent review articles have been written on the subject, a selection is given in Ref. [3].

Some applications of MWPCs may be cited as crystal diffraction, beta chromatography and dual energy angiography. A low dose X-ray digital radiography scanner based on the MWPC (see fig. 1) was invented by Shekhtman, [4] and is presently being applied routinely to examine patients in hospitals in Russia and France [5,6]. Fig. 2 taken from [6] shows a follow-up film of congenital hip dislocation (Perthes disease) in a 7-year-old boy with satisfactory visualization of the femoral architecture and bone texture. Upgrades of this device for improved resolution and dose reduction are still in progress $[6,7]$.

\footnotetext{
${ }^{2}$ Presently at CERN, CH 1211, Geneva, Switzerland. E-mail Archana.Sharma@ cern.ch
} 


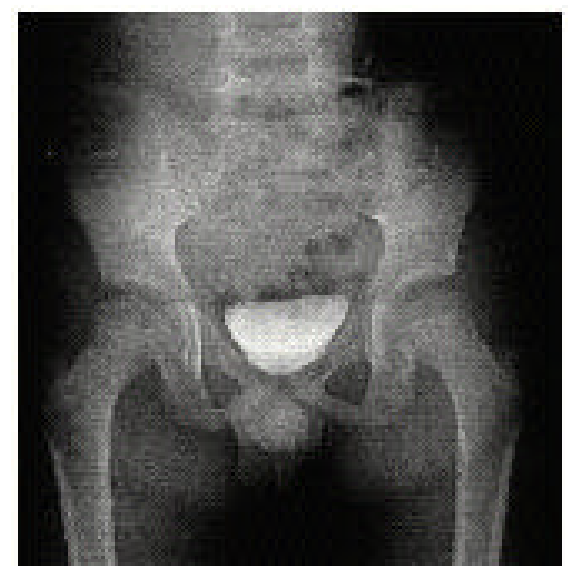

Fig. 2 Digital Radiography of a child using MWPC Scanner

Despite their obvious success, some fundamental limitations of multiwire proportional chambers restrict their use for high rate application. Detailed discussions of these characteristics may be found in the cited literature: the wire spacing limits the position accuracy and two track resolution to $\sim 1 \mathrm{~mm}$. The electrostatic instability limits the stable wire lengths; the widths of the induced charges limit the pad response function, and most importantly at high rates, accumulation of positive ions restrict the rate capabilities of MWPCs.

The advent of high luminosity colliders [8] demands fast, highly performing position sensitive detectors. Ever more stringent, unsurpassed position, two track, two dimensional and time resolutions, ability to operate withstanding long term radiation and background environment are key requirements.

The invention of the Micro-Strip gas Chamber (MSGC) by Oed [9] ushered another new era of gaseous detectors. A recent review [10] summarizes in detail the MSGCs and its derivatives, here I will briefly mention their key features and some applications.

\section{THE MICRO STRIP GENERATION}

An MSGC consists of a pattern of thin anodes and cathode strips laid on an insulating substrate with a pitch of a few hundred $\mu \mathrm{m}$. Delimited by a drift electrode above and appropriate potentials applied, the resulting electric field ${ }^{3}$ is as shown in Fig. 3. The design itself removes the positive ions from the vicinity of the avalanches, thereby lending

\footnotetext{
${ }^{3}$ All electric fields and simulations presented in this work are taken from Ref. [10] or are unpublished work of the author.
}

high rate capability to this device almost two orders of magnitude higher $\left(\sim 10 \% \mathrm{~mm}^{2}\right)$ than MWPCs $\left(\sim 10^{4} / \mathrm{mm}^{2}\right)$.

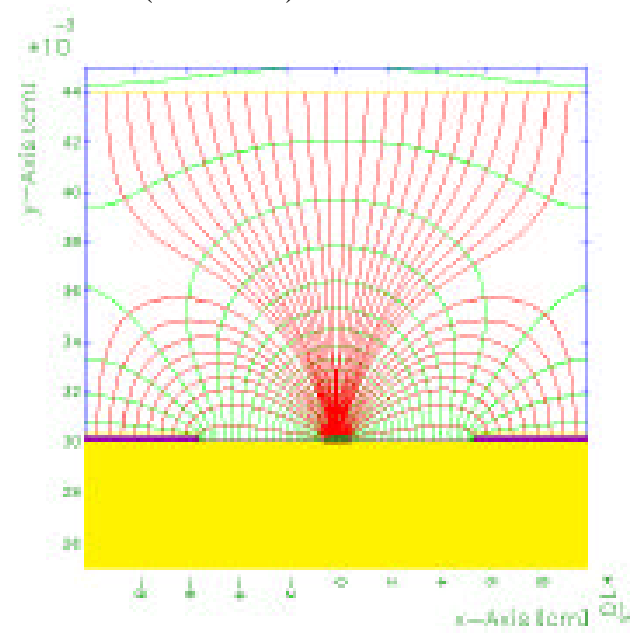

Fig. 3 Field Configuration in an MSGC with typical voltage settings

The salient features of its operation are localization accuracies $\sim 30 \mu \mathrm{m} \mathrm{rms}$, double track resolution of $400 \mu \mathrm{m}$, and good energy resolution. Long term and magnetic field operations have been demonstrated and, apart from high energy physics [11] these devices have found applications in many varied fields of X-ray spectrometry and digital radiography.

The difficulties associated with operating MSGCs began [12] when they were exposed to highly ionizing particles usually present in a high luminosity machine backgrounds from low energy $\gamma$ 's, proton conversions and nuclear fragments. The highly ionizing particles deposit almost three of magnitude more charge in the detection volume as compared to a minimum ionizing particle. Charging up of surface defects, long lived excited states and overlapping avalanches seem to be the culprit lowering the discharge limits of operation of these devices; several groups dedicatedly worked to examine and understand these defects [13-17]. What emerges is that the streamer mode of operation in the case of an MWPC is stable due to the fact that the el

ectric field in the direction of the propagation of the streamer is increasingly weak (between the anode and cathode). In case of micro-strip detectors, the anode-cathode distance is very small $(\sim 50-100 \mu \mathrm{m})$, electric field at the tip of the streamer and that along the surface being high, the streamer is most likely to be followed by a, voltage and ionization density dependent, discharge. With this insight, several novel designs appeared on 
the horizon, some of which are discussed below.

\section{THE 'NEW MICROPATTERN' ERA}

\section{A. MICRO-DOT AND MICRO-MEGAS}

Advances in photolithography and application of silicon foundry techniques heralded a new era in the design and fabrication of 'Micropattern Detectors'. The Microdot detector ( $\mu$ DOT) [18] invented by Biagi is the ultimate gaseous pixel device with anode dots surrounded by cathode rings. With very high gains $\sim 10^{6}$, it does not discharge [19], probably due to the fact that the field emulates the $1 / \mathrm{r}$ field drop just like that from an anode wire.

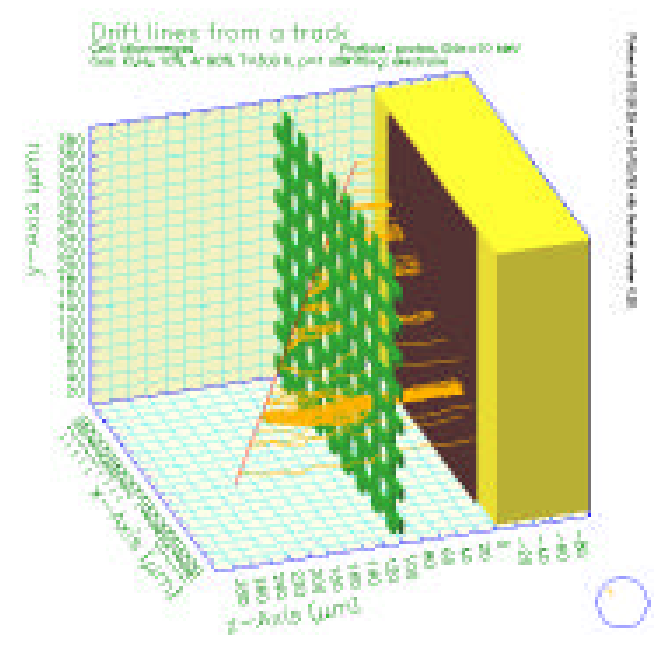

Fig. 4

A very asymmetric parallel plate chamber [20], the MICROMEGAS detector invented by Charpak and Giomataris [21], takes advantage of the semi-saturation of the Townsend coefficient at high fields $\sim 100 \mathrm{kV} / \mathrm{cm}$ in several gas mixtures [22], thus being stable in its operation with minimum ionizing particles. Fig. 4 shows electrons drifting from the sensitive volume into the amplification volume. Large area, $40 \mathrm{x} 40 \mathrm{~cm}^{2}$ MICROMEGAS detectors are being made and tested [23] for the COMPASS experiment at CERN.

\section{B. CATs}

A new kind of detector was invented by Lemonnier et al during the same era (19951996) as the above detectors, called the CAT or 'Chambre A Trous' ${ }^{4}$ [24]. It consists of a narrow hole micro-machined in an insulator metallized on the surface as the cathode, the metal at the bottom of the hole constitutes the anode. With appropriate potentials and a drift electrode, this scheme acts as a focussing lens for the drifting electrons left in the wake of an ionizing radiation. Removing the insulator in between leaves the cathode as a micro-mesh, which when placed with a thin gap above the readout electrode, emulates the CAT operation hence named microCAT or $\mu \mathrm{CAT}$, see Fig. 5 ( $a$ and $b$ ) [25]. This structure offers gains of several $10^{4}$. With an ingenious scheme of readout from 'virtual pixels' made by current sharing, offering 20 times finer resolution and 400 times more virtual pixels, see Fig. 5(b) the $\mu \mathrm{CAT}$ combined with the VIrtual pixels is renamed the VIP [26].

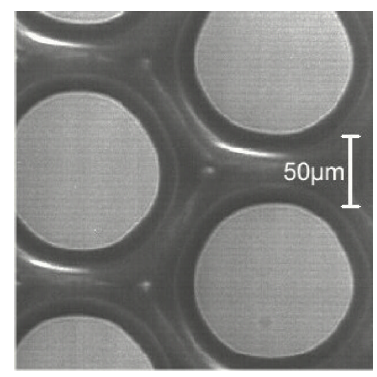

Fig. 5 (a)

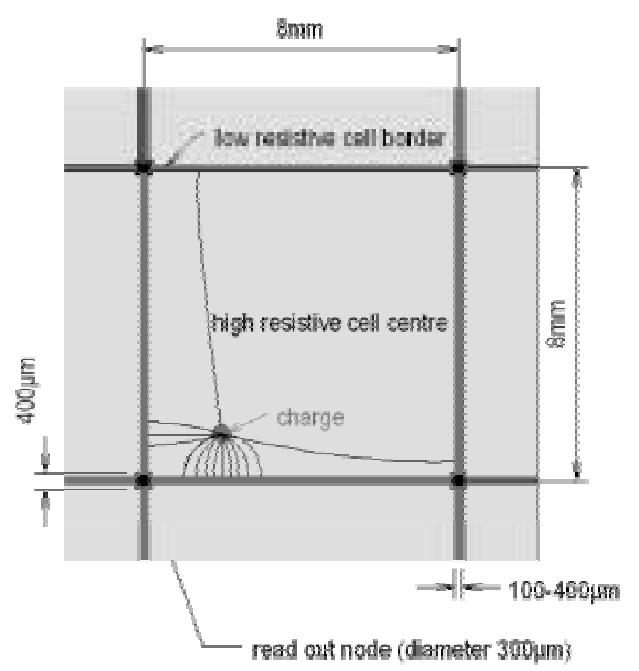

Fig. 5 (b )

\section{GEMs}

A new concept of gas amplification was introduced in 1996 by Sauli, with the Gas Electron multiplier (GEM) [27], manufactured

\footnotetext{
${ }^{4}$ Chamber or detector made of holes $(F$.)
} 
by using standard printed circuit wet etching techniques $^{5}$. Comprising a thin $(\sim 50 \mu \mathrm{m})$ Kapton foil, double sided clad with Copper, holes are perforated through and the two surface maintained at a potential gradient, thus

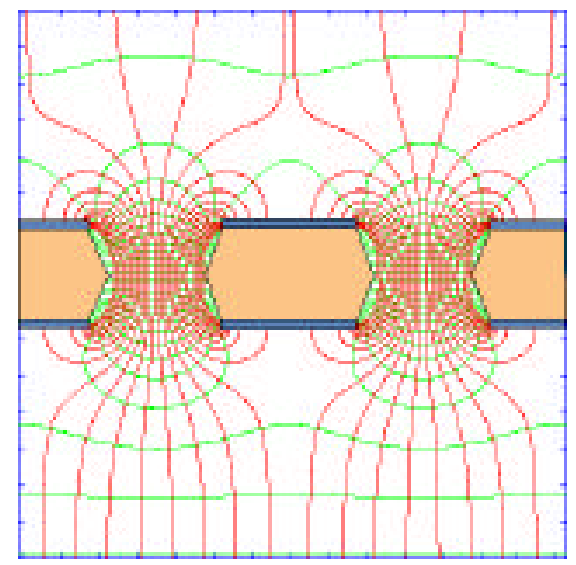

Fig. 6(a)

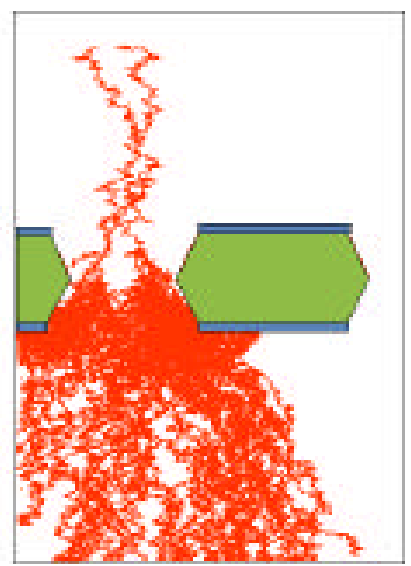

Fig. 6 (a above) Field across a GEM, and (b) An electron avalanche in the GEM channel.

providing the necessary field for electron amplification, as shown in fig. 6(a), and an avalanche of electrons as in Fig. 6(b). Coupled with a drift electrode above and a readout electrode below, it acts as a highly performing micropattern detector. The essential and advantageous feature of this detector is that amplification and detection are decoupled, and the readout is at zero potential. Permitting charge transfer to be coupled to a second amplification device, this opens up the possibility of using a GEM in tandem with an MSGC or a second GEM.

\footnotetext{
${ }^{5}$ At the Printed Circuit \& Surface Treatment Workshop at CERN, Geneva, Switzerland
}

\section{More Micropatterns}

With this technique and following the GEM concept and better understanding of the discharge phenomena, several new 'micro'detectors have appeared on the scene: MicroWire [29], an extension of the $\mu$ DOT in the third dimension Micro-Pin Array (MIPA) [30], the Micro-Tube [31], Micro Well [32], Micro Trench [33] and Micro Groove [34]. All of these authors have tried to minimize the presence of insulators in between the anode and cathode - the culprit for gliding discharges along the surface. Fig. 7(a) shows a microphotograph of the Micro-Tube detector [ ], with a field map in fig. 7(b) [35]. Fabricated using combination of laser micro-machining and nickel electroplating it consists of $\sim 150$ $\mu \mathrm{m}$ diameter cathode, and an anode tube, which is machined through the well and plated alongside.

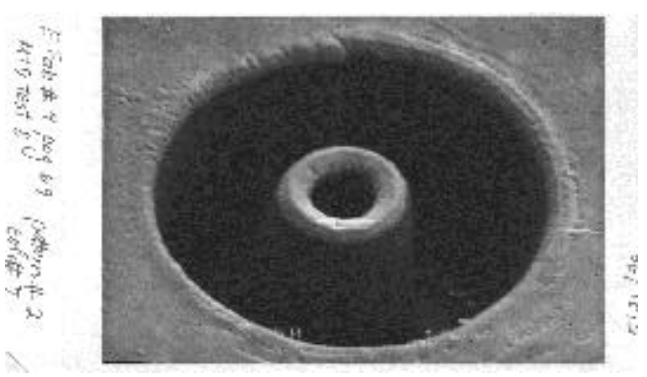

Fig. 7(a) The Microtube: the central pin is metallized on the outside, emulating an anode wire.

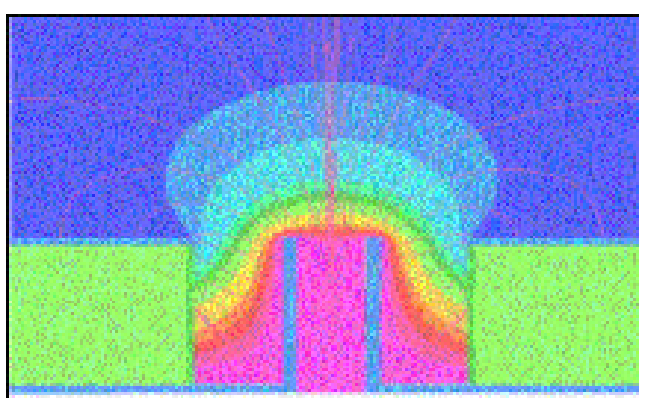

Fig. 7(b) Field across a Microtube

This structure results in an electric field that increases rapidly at the anode, similar to the $\mu$ DOT. Unlike the $\mu$ DOT, however, there is no insulating material on the direct line of sight from the cathode to the anode. These design features are predicted to lead to higher gas gains, better stability with fewer discharges, and the reduction of charging effects. An investigation of the effects of detector geometry upon Microtube performance shows similar performance to the 
$\mu \mathrm{DOT}$ and $\mu \mathrm{CAT}$ detectors, it also offers large gains $\sim 10^{4}[36]$.

As mentioned above, detailed studies have shown that discharges in the presence of highly ionizing particles appear in all micropattern detectors at gains of a few thousand [19]. It is possible to obtain higher gains with poorly quenched gases, since they permit a lower operating voltage, and have a higher diffusion, thus lowering the charge density and photon feedback probability. Combining the MSGC with a GEM safe operation has been demonstrated up to gains of few ten thousands; 200 large size such detectors are operating at HERA-B [37]. The DIRAC [38] experiment at the CERN PS also employs MSGC + GEM detectors, which have permitted to improve the momentum resolution by a factor of two; Fig. 7 shows an assembled GEM + MSGC, and a fine example of vertex reconstruction using this detector.
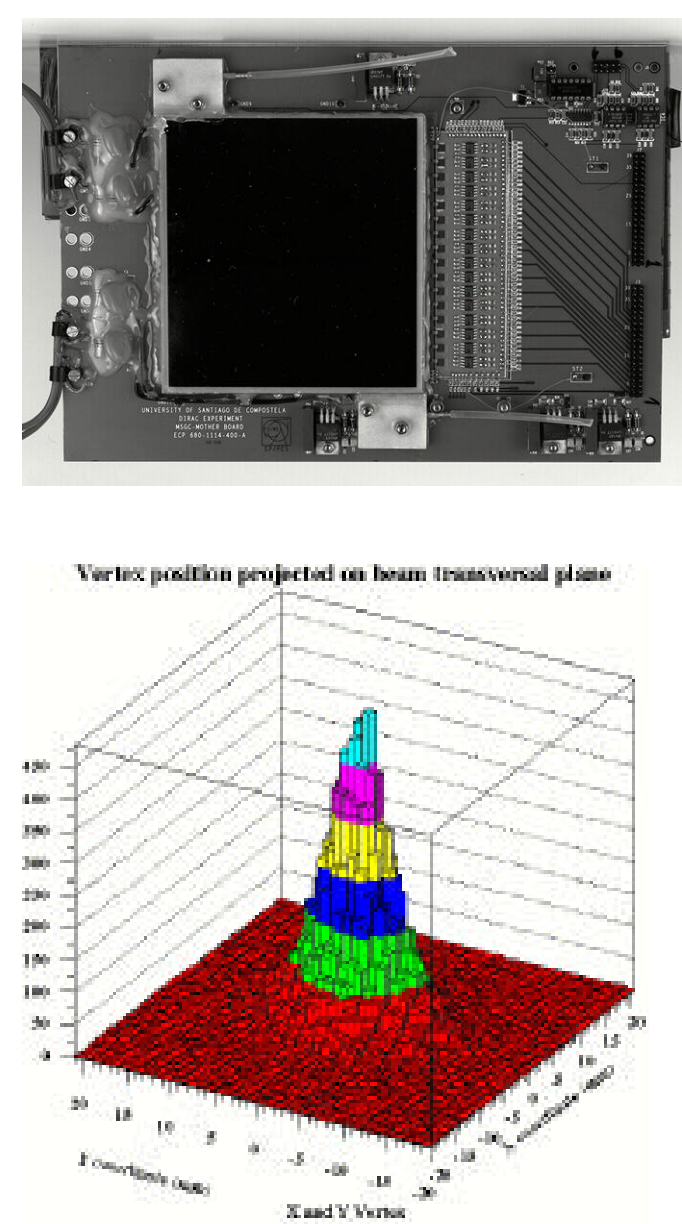

Fig. 8 (a) (above) An MSGC + GEM assembly for DIRAC tracker, and (b) Vertex reconstruction.

Putting two GEMs in tandem offers a robust detector, which has been studied in detail [39], and large sizes are being built for the COMPASS experiment [40], Carrying the concept further adding a third GEM offers an even more stable operation in worst hadronic beam environment as demonstrated in Ref. [41]. At gains $\sim 10^{4}$, spark probabilities $\sim 10^{-10}$ have been measured ${ }^{6}$. Fig. 9 shows the probabilities of discharges in a single, double and triple GEM detector [42].

For large sizes, the GEMs are segmented in order to reduce the capacity, thus limiting the energy in a discharge.

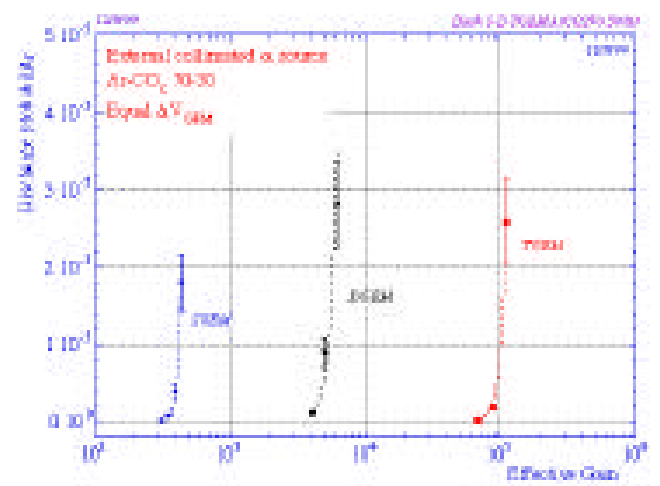

Fig. 9 Probability of a discharge, normalized to the number of heavily ionizing tracks, versus gain for single, double and triple GEM detectors.

\section{POTENTIAL APPLICATIONS OF THE 'MICRO-GENERATION'}

\section{MSGCs for X-ray Imaging}
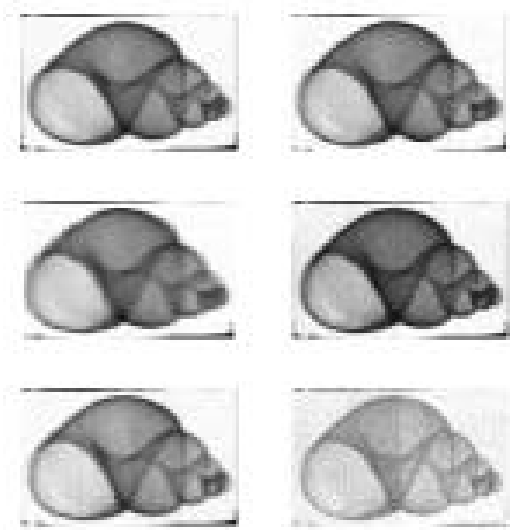

Fig. 10 Images of a snail shell taken with an MSGC operating with Xe at 4 bar [43].

Conventional film radiography has very good spatial resolution but limited dynamic range. Digital scanned projection radiography offers improved dynamic range and adjustable

\footnotetext{
${ }^{6}$ See refs. $[13,29,33]$ for definition of spark probabilities
} 
contrast level. The image can be further enhanced using photon energy information. This has been made possible by using the MSGCs with pure Xe at high pressures; an example of an image is shown in fig. 10.

\section{B. TPC readout}

For the TESLA experiment at the future Linear Collider [44] a double or triple GEM configuration is under consideration [45] owing to its fast electron signal, minimal magnetic distortion effects, and suppression of ion feedback by design. Fig. 11 shows some measurements and simulation of the fractional positive ion feedback in a double GEM [46].

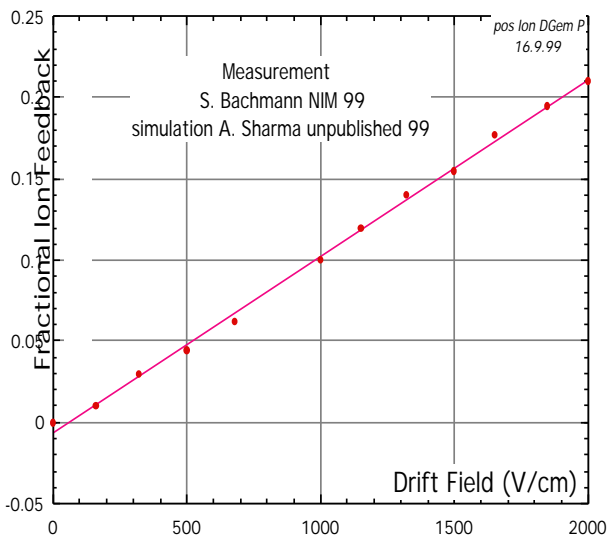

Fig. 11

Special hexagonal pads are being developed [47] proving unprecedented 50 to $60 \mu \mathrm{m}$ resolution in a TPC using charge sharing and induction signals.

\section{The MICROMEGAS X-ray Gallery}

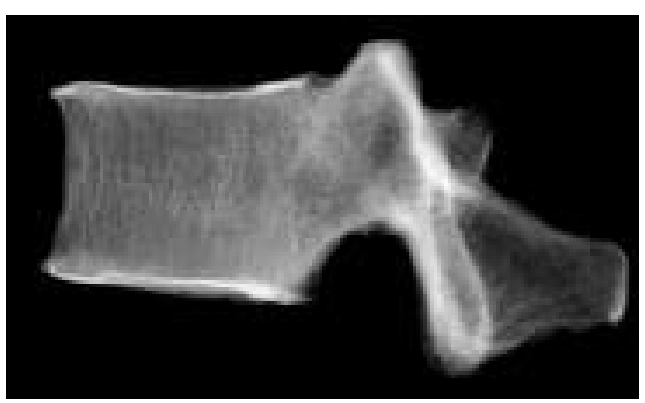

Fig. 12

Operating in pure Xenon at atmospheric pressure, the MICROMEGAS detectors have been developed for X-ray imaging. An example shown in Fig. 12 [48], of a vertebra scanned with a MICROMEGAS.
D. Protein Crystallography SAXS (Small Angle $X$-ray Scattering)

X-ray diffraction studies using MSGCs have yielded rapid analysis of single crystal structures using the information of position and time of the incident X-rays: crystal structures of organic molecules can be obtained in a matter of minutes [49],. Fast time resolved X-ray diffraction measurements offer a time variation of the SAXS pattern of a protein solution for example, shown in fig. 13, within a frame time of $10 \mathrm{~ms}$.
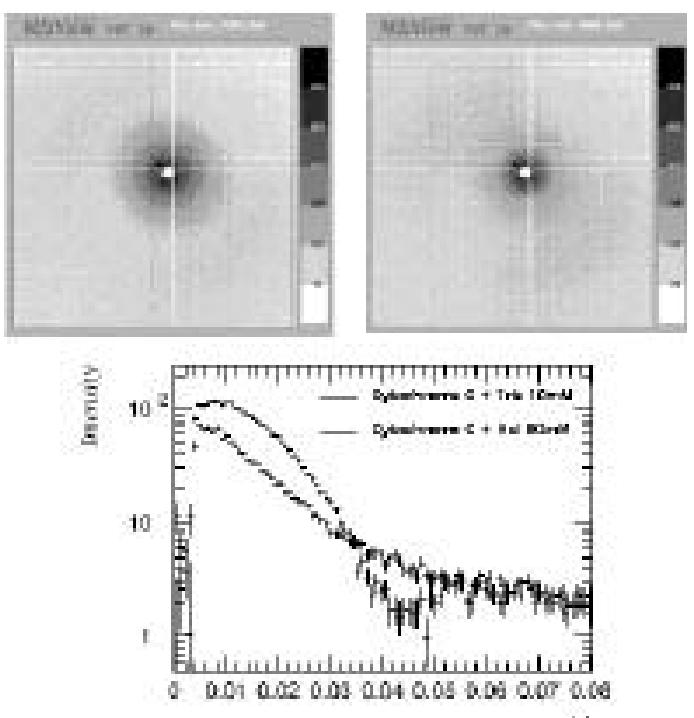

is

Fig. 13 Above: X-ray diffraction patterns of Cytochrome $\mathrm{C}$ different concentrations of contamination molecules. Below: X-ray diffraction intensities of the same, measured more than a minute after mixing the solutions.

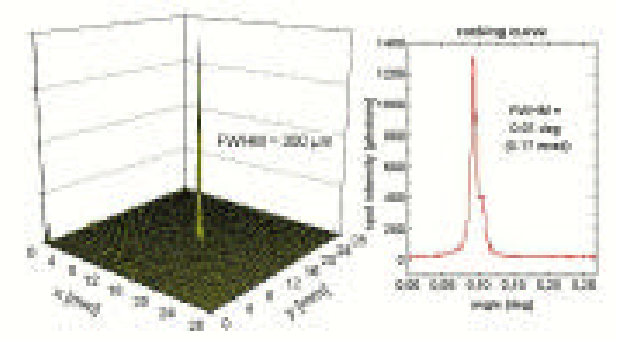

Fig. 14

A diffraction pattern of a lipid membrane is shown in fig. 14, made with the VIP detector (see section III.B above) at Elettra, a synchrotron source in Trieste, Italy. With complex algorithms, especially made for the (VIP) readout cell border, and superimposing 
several shots of images a high degree of detail may be obtained from diffraction patterns: fig. $15[50]$.
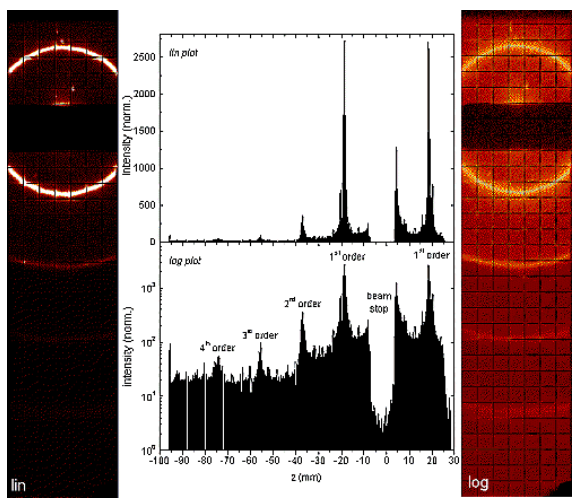

Fig. 15

\section{E. Digital mammography $U V$ and Visible Photomultipliers using GEM}

A combination of several GEMs and its real time two-dimensional localization capabilities shows great promise for an application to digital mammography [51][?] The essential features are a large flat area and high resolution. With a photocathode (UV, visible) couple to a micropattern detector, sealed gas avalanche photomultipliers are being developed for fast imaging of UV and visible light, flat readout devices for scintillator and scintillating fiber arrays, and as medical imaging. Photocathodes have also been attempted to be combined with the Glass Capillary Plate (GCP) detectors [52], with advantages of reduced photon photon feedback and the high level of cleanliness necessary for the manufacture of high efficiency of photocathodes/secondary photon emitters.

To this end, single photon detection has been actively pursued using three and four GEMs in tandem, and very large gains $\sim 10^{5}$ have been obtained in pure noble gas (Ar), and $10^{6}$ with an admixture of few percent $\mathrm{CH}_{4}$ [53]. With a small preamplification in the drift region, combined with high diffusion, fully efficient single photon detection is predicted and measured [54,55] as demonstrated in figs. 16 ( $a$ and b). Fig. 17 shows an example of a large signal in pure Argon with a three GEM combination [56].

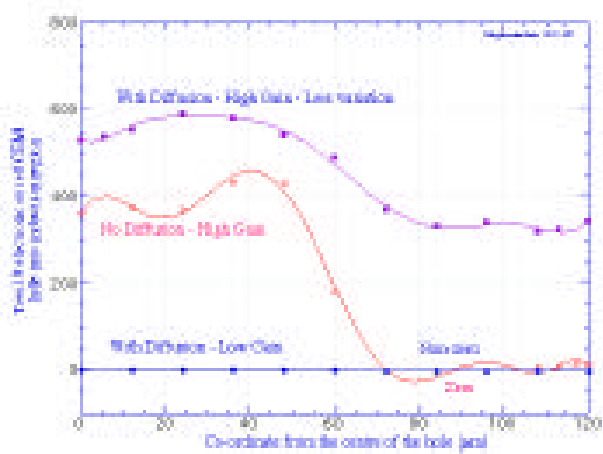

Fig. 16 (a) Transmission of electrons in a GEM

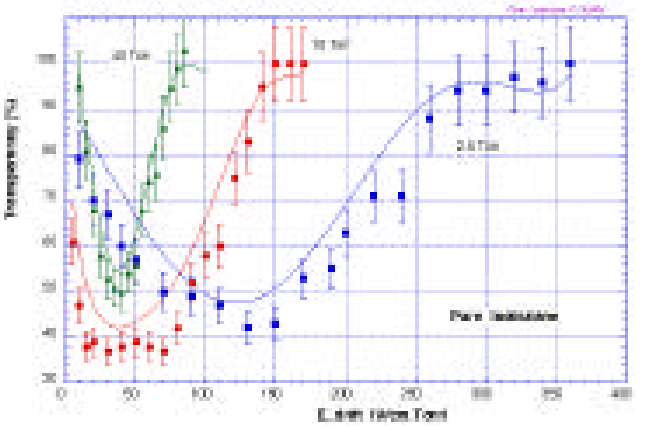

Fig. 16(b) Full Efficiency in a GEM with a transmissive photocathode and preamplification in the drift region.[]

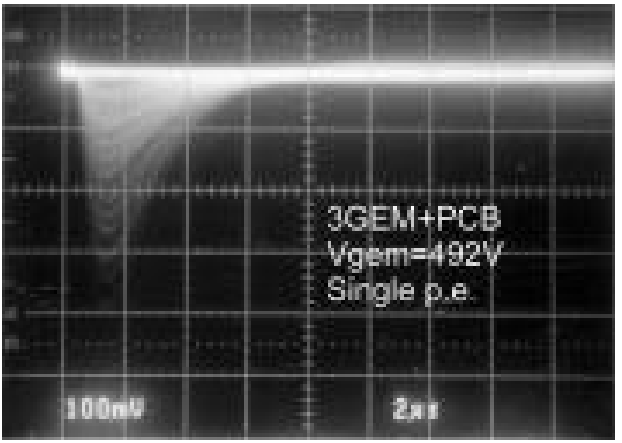

Fig. 17 Signals with a Triple GEM

\section{F. Cherenkov Ring Imaging (RICH/DIRC)}

A feasibility study aimed at improving the detection of photons emitted by Cherenkov light, for example for an upgrade of existing CRID [56], at SLAC was made. Using a cascade of four GEMs and an operating gas as pure ethane, very high gains have been observed [57], as shown in fig. 18. 


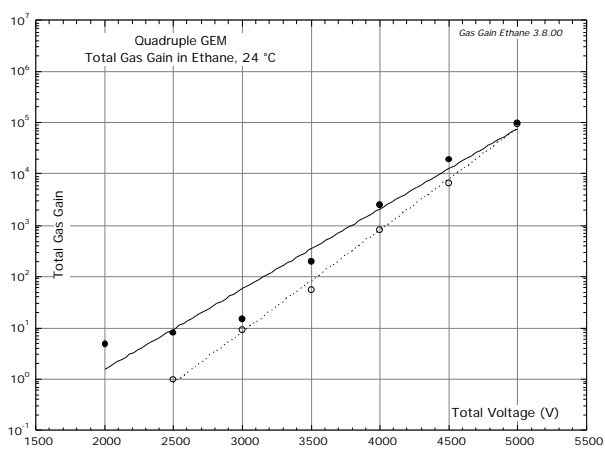

Fig. 19 Gain in a Quadruple GEM cascade

\section{G. Scintillation Light Imaging}
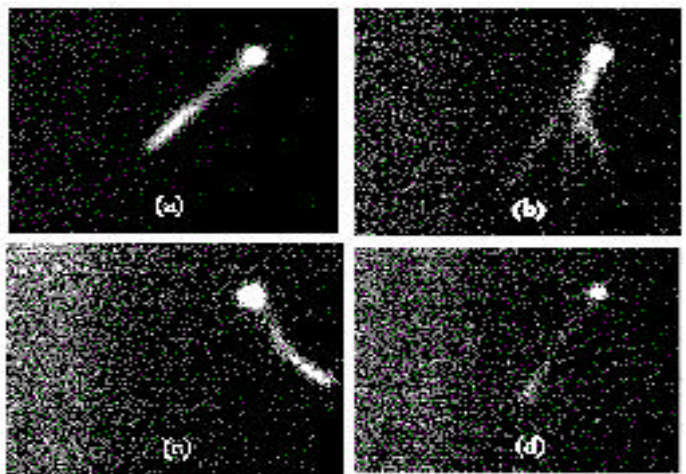

Fig. 20 Scintillation images of alpha tracks in $\mathrm{Ar}^{-\mathrm{CF}_{4}}$

With a GEM as amplifier and a CCD camera, X-ray images of individual projected alpha tracks are visible using the scintillation properties of $\mathrm{CF}_{4}$, as shown in Fig. 8 [58].

\section{H. X-ray imaging: Radiology and diagnostics}

With a GEM + MSGC combination operating in $\mathrm{Xe}^{-} \mathrm{CH}_{4}$ at 4 atm the following Xray images have been taken as an excellent example of imaging for diagnostics with a micropattern detector [59],
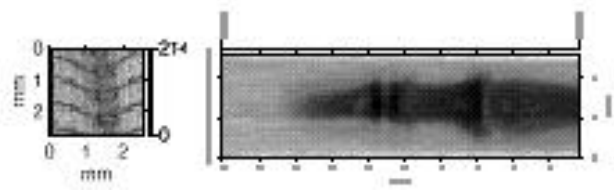

Fig. 21. (Left) A $3 \mathrm{~mm} \times 3 \mathrm{~mm} 13 \mathrm{kV}$ X-ray absorption radiography of a fish bone taken at $2 \mathrm{~atm}$. Right: a $3 \mathrm{~mm} x$ $10 \mathrm{~mm} 50 \mathrm{kV}$ x- ray image of a digit of a mouse.

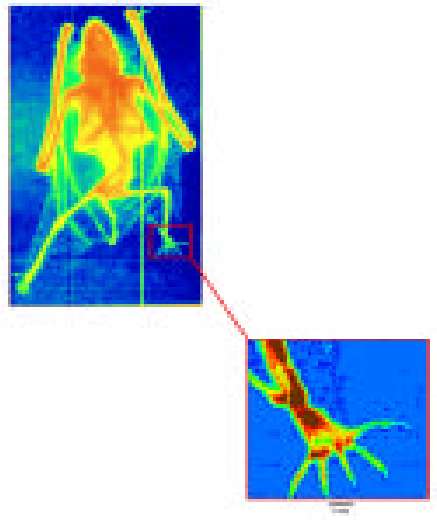

Fig. 22 Radiography of a micromammal using GEM and $50 \mu \mathrm{m} \times 50 \mu \mathrm{m} 2 \mathrm{~d}$-readout []

Specialized two-dimensional readout boards have been manufactured using the GEM technology. Operating at ground potential, these boards in conjunction with GEMs have been developed for digital absorption radiography [60]. With a pixel size of $50 \mu \mathrm{m}$, the image of a micromammal (bat, width $32 \mathrm{~mm}$ ) is shown in fig. taken from [].

\section{Imaging of Polarized X-rays.}

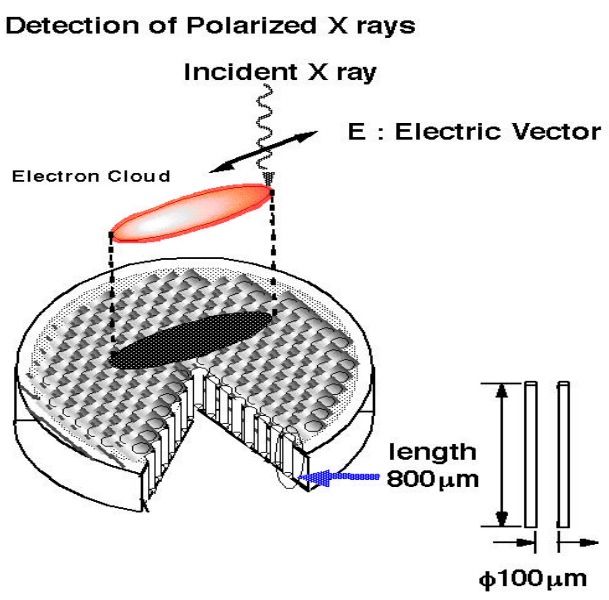

Fig. 23 Detection of polarized photons with a Glass Capillary Imaging Gas Proportional Counter

In X-ray astronomy, measurements of Xray polarization are useful to investigate features of magnetic fields such as pulsars, synchrotron nebulae, and so on. Some X-ray polarimeters have been developed using GCPs [61],. 


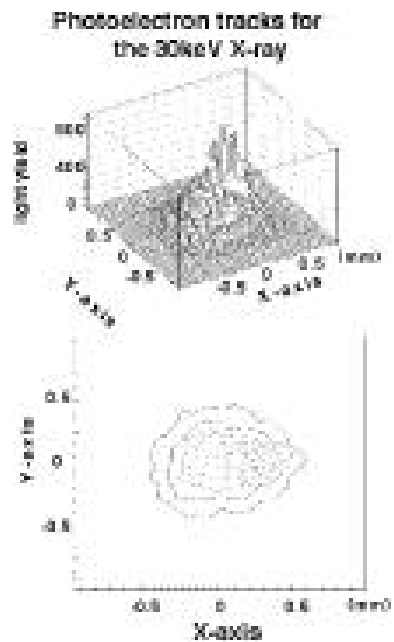

Fig. 24 Photoelectrons from a GCP Polarimeter

Because the emission direction of the primary electron depends on the polarization of the incident $\mathrm{X}$-rays, the information on the polarization can be deduced, acquiring the information on the shape of electron clouds. The performance of the Polarimeter is expressed as the function of detection efficiency and modulation factor, and hence these parameters depend on gas pressure and gas depth. Fig. (24) shows some images of tracks from with a GCP [61].

\section{J. GEM for Plasma Diagnostics}

Exploiting the selective sensitivity of the GEM to soft X-rays, imaging the dynamics of a plasma has been attempted by the Frascati and Pisa group $[62,63]$ for the (FTU) Frascati Tokamak Upgrade [64]. With a GEM and individual pixel readout, time resolved plasma diagnostics are made giving information about temperature and turbulence effects. Fig. 10 shows a reconstruction of a photoelectron released near the center of the detector.

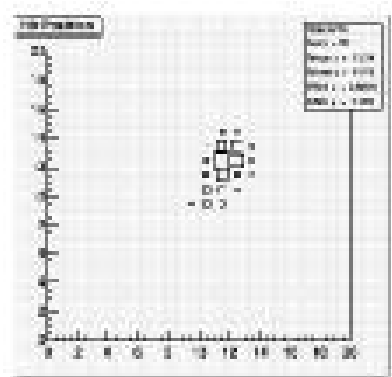

Fig. 25.(a)

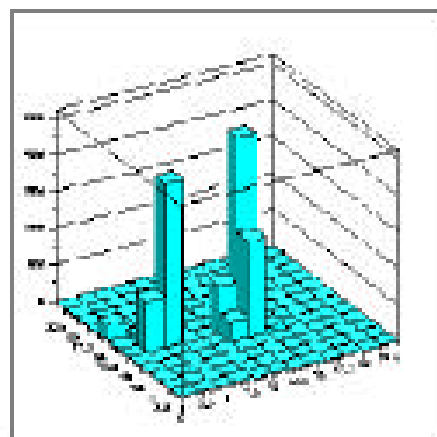

Fig. 25 (b)

\section{CONCLUSIONS AND OUTLOOK}

Multiwire chambers have matured since their introduction over the last few decades, with svereal applications in particle physics and diagnostics of various kinds. The last decade has seen several novel developments in Micropattern Gaseous Detectors of which some are summarized in this overview. Basic understanding of the discharge mechanisms in these devices has also improved allowing amelioration of their design. Progress in manufacture of customized readout boards has evolved revolutionizing the potential applications of these detectors in radiology, diagnostics, astrophysics and other fields.

\section{REFERENCES}

[1] Phil. Mag. Xiii(1896)392; Conduction of electricity through gases (Ist ed, 1903);

Proc. Of Royal Society. A81(1908)141

[2] G. Charpak et al Nucl. Instr. And Meth. 62(1968)262-268

[3] MWPC Reviews

[4] E. Babichev et al (1992) Nucl Instrum Methods Phys Res A323: 49, and references therein, S. Baru eta al Novosibirsk Preprint 35(1989) 98-39

[5] E. Babichev et al Elba 2000 \& Porosev Priv. Comm. Oct 2000

[6] Martinez-Davalos A, Speller RD, Horrocks JA, et al (1993) Evaluation of a new low-dose digital $x$-ray system. Phys Med Biol 38: 1419 \pm 1432

[7] G. Kalifa et al, Pediatric. Radiology 28(1998)557

[8] Large Hadron Collider (LHC) @ CERN, Geneva, Switzerland, B-factories at SLAC and KEK.

[9] A. Oed, Nucl. Instr. And Meth. A263(1988)35 
[10] F. Sauli and A. Sharma, Annual Rev. of Nucl. Sci. 49(1999)41

[11] R. Bouclier et al CMS-TN/96-018 (1996)

[12] B. Boimska et al, CMS-TN/97-020

(1997); Nuclear Physics B 61B (1998) 498;

[13] B. Schmidt, Nucl. Instr. And Meth.

A419(1998)230

[14] V. Peskov et al, IEEE Trans, Nucl. Sci.

NS-45:244(1998)

[15] A. Bressan et al Nucl. Instr. And Meth. A 424(1998)321

[16] P. Fonte et al Nucl. Instr. And Meth. A 419(1998) 405

[12] S.F. Biagi et al, Nucl. Instrum. Methods A371(1995)12

[13] A. Bressan, A. Buzulutskov, L. Ropelewski, F.Sauli and L. Shekhtman, Nucl. Instr. And Meth. A 432(1999)119-124

[14] Asymmetric PPAC

[15] Y. Giomataris et al Nucl. Instr. And Meth. A 376(1996)29

[16] A. Sharma, ICFA Bulletin Fall 1999 http://www.slac.stanford.edu/pubs/icfa

[17] F. Kuune Elba 2000

[18] G. Charpak and M. Meynadier Priv. Com. Oct. 2000

[19] F. Bartol et al., J. Phys. III France 6 (1996) 337

[20] A. Sarvestani et al Nucl. Instr. And Meth. A 419(1998)444

[21] N. Pavel, Siegen, SPIE ? Priv. Com. Oct 2000

[22] F. Sauli Nucl. Instr. And Meth. A 386(1997)531

[23] B. Adeva $\mu$ Wire

[24] P. Rehak et al MIPA

[25] Well Pitts

[26] $\mu \mathrm{WELL}$

[27] Trench

[28] $\mu$ Groove R. Bellazzini

[29] V. Peskov IEEE 2000 contribution to this conference

[30] C. Richter, PhD. Thesis October 2000 submitted to University of Heidelberg, Germany.

[31] DIRAC

[32] J. Benlloch et al, IEEE trans NUCl. Sci. NS-45(1998) 234

J. Benlloch et al NIM A 419 (1998) 410

[33] LHB Straumann

[34] A. Bressan et al Nucl. Instr. And Meth. A 425(1999)254, 262

radiography Bressan + Madhu

[35] TESLA Linear Collider @ DESY Hamburg

[36] M. Dixit P. Com Carleton DESY Karlsruhe

[37] A. Sharma, To appear in Nucl. Instr. and Meth. as Proceedings of Symposium on Applications of Particle Detectors in Medicine,
Biology and Astrophysics, 5-8 October 1999, Siegen, Germany, also as CERN-OPEN99/373.

[38] F. Fraga, Contribution to this Conference [39] DIGITAL MAMO Project MICADO

[40] A. Buzulutskov et al Subm. Nucl. Instr. And Meth. (1999), A. Buzulutskov, L. Shekhtman, A. Bressan, A. Di Mauro, L. Ropelewski, F. Sauli and S. Biagi, Nucl. Instr. And Meth. A 433(1999)471-475

[41] A. Sharma CERN Preprint 99/372, Submitted for publication to Nucl. Instr. \& Meth. (1999)

[42] C. Richter et al To Appear in the Proceedings of Frontier Detectors for Frontier Physics, Elba 2000.

[43] A. Buzulutskov, Contribution to this conference

[44] G. Spandre Priv. Com Oct. 2000

[45] A. Pacella

[46] Frascati Tokamak Upgrade 2000 